\title{
Supersymmetric dark matter and the reheating temperature of the universe
}

\author{
N. Fornengo* \\ Dipartimento di Fisica Teorica, Università di Torino, Istituto Nazionale di Fisica Nucleare, Sezione di Torino, via P. Giuria 1, \\ I-10125 Torino, Italy \\ A. Riotto ${ }^{\dagger}$ \\ Istituto Nazionale di Fisica Nucleare, Sezione di Padova, via F. Marzolo 8, I-35131 Padova, Italy \\ S. Scopel \\ Dipartimento di Fisica Teorica, Università di Torino, Istituto Nazionale di Fisica Nucleare, Sezione di Torino, via P. Giuria 1, \\ I-10125 Torino, Italy \\ (Received 26 August 2002; published 29 January 2003)
}

\begin{abstract}
Since the thermal history of the universe is unknown before the epoch of primordial nucleosynthesis, the largest temperature of the radiation-dominated phase (the reheating temperature) might have been as low as 1 $\mathrm{MeV}$. We perform a quantitative study of the supersymmetric dark matter relic abundance in cosmological scenarios with low reheating temperature. We show that, for values of the reheating temperature smaller than about $30 \mathrm{GeV}$, the domains of the supergravity parameter space which are compatible with the hypothesis that dark matter is composed of neutralinos are largely enhanced. We also find a lower bound on the reheating temperature: If the latter is smaller than about $1 \mathrm{GeV}$ neutralinos cannot be efficiently produced in the early universe and then they are not able to explain the present amount of dark matter.
\end{abstract}

DOI: 10.1103/PhysRevD.67.023514

PACS number(s): 98.80. $-\mathrm{k}, 95.35 .+\mathrm{d}$

\section{INTRODUCTION}

Supersymmetric dark matter provides one of the hottest topics on the border between cosmology and particle physics. This is due to the fact that in $R$-parity conserving supersymmetric theories the lightest supersymmetric particle (LSP) is stable and may provide the cold dark matter, whose existence is inferred from a large number of independent observations [1,2]. Among the different supersymmetric candidates, the neutralino turns out to be a perfect dark matter particle, since it has neither charge nor color, its only interactions being of the weak type.

The present abundance of neutralinos depends on the thermal history of the universe. In the early universe interactions may keep neutralinos in thermal equilibrium with the radiation bath until their abundance freezes out at a temperature $T_{F}$. The neutralino mass is constrained by accelerator data to be heavier than a few tens of GeV. This implies that it decouples in the early universe when it is nonrelativistic, at $T_{F}$ in the $\mathrm{GeV}$ range. This picture is correct if the maximum temperature in the radiation-dominated era, which from now on we will refer to as the reheating temperature $T_{R H}$, is much larger than the freeze-out temperature $T_{F}$. If this is the case, the neutralino relic abundance turns out to be

$$
\Omega_{\chi} h^{2} \equiv \frac{\rho_{\chi}}{\rho_{c}} h^{2} \propto \frac{2.6 \times 10^{-10} \mathrm{GeV}^{-2}}{\left\langle\sigma_{\mathrm{ann}} v\right\rangle}
$$

where $\rho_{c} \equiv 1.8791 h^{2} \times 10^{-29} \mathrm{~g} \mathrm{~cm}^{-3}$ is the critical density, $h$

\footnotetext{
*Electronic address: fornengo@to.infn.it

†Electronic address: antonio.riotto@pd.infn.it

¥Electronic address: scopel@to.infn.it
}

is the Hubble constant in units of $100 \mathrm{~km} \mathrm{sec}^{-1} \mathrm{Mpc}^{-1}$, while $\sigma_{\text {ann }}$ is the weakly interacting massive particle (WIMP) pair-annihilation cross section, $v$ is the relative velocity between the two annihilating particles, and angular brackets denote the thermal average. Indeed, what specifically makes the neutralino an ideal dark matter candidate is that in Eq. (1) the value of the annihilation cross section, calculated in a wide variety of supersymmetric (SUSY) models, may yield a result for $\Omega_{\chi} h^{2}$ which falls in the correct interval suggested by present day observations for the amount of nonbaryonic dark matter in the universe $[1,2]$ :

$$
0.05 \leqq \Omega_{\mathrm{M}} h^{2} \lesssim 0.3 .
$$

When exploring the neutralino parameter space and its chances of discovery in both accelerator and nonaccelerator searches, this argument has usually been turned the other way around, and Eq. (2) used as a constraint on the neutralino parameter space. Depending on the particular supersymmetric scenario, this may have important consequences on the allowed supersymmetric configurations. In particular, in supergravity- (SUGRA-)inspired models, the allowed neutralino parameter space turns out to be severely reduced by requiring that $\Omega_{\chi} h^{2}$ falls inside the interval defined by Eq. (2) [3-13].

The robustness of these constraints relies on the cosmological assumptions that lead to Eq. (1). Indeed, the thermal history of the universe before the epoch of nucleosynthesis is unknown. The maximum temperature in the radiationdominated era $T_{R H}$ may have been as low as $1 \mathrm{MeV}$ (but not smaller in order not to spoil the nucleosynthesis predictions). The possibility of a low reheating temperature of the universe has recently been discussed in Ref. [14]. There it was shown that a low reheating temperature has important impli- 
cations for many topics in cosmology such as axion physics, leptogenesis, and nucleosynthesis constraints on decaying particles. In particular, it was shown that stable weakly interacting massive particles may be produced even if the reheating temperature is much smaller than the freeze-out temperature of the dark matter particles, $T_{R H}<T_{F}$, and that the dependence of the present abundance on the mass and the annihilation cross section of the dark matter particle may differ drastically from standard results. ${ }^{1}$

The goal of this paper is twofold: First, we wish to extend the analysis of Ref. [14] and perform a quantitative study of the case of neutralinos in SUGRA scenarios, analyzing in detail the impact that a low $T_{R H}$ may have for the present neutralino relic abundance; secondly, we aim at providing a lower bound on the reheating temperature. The logic is the following. All matter is produced at the end of inflation [17] when all the vacuum energy stored into the inflaton field is released and the universe becomes radiation dominated with the initial temperature $T_{R H}$. During the reheating process, particles are generated through thermal scatterings and quickly thermalize. Among them, dark matter particles may also be produced but their final number depends strongly on the reheating temperature. If the latter is too small, the thermal bath does not give rise to a number of neutralinos large enough to make them good candidates for dark matter. This leads to a lower limit on $T_{R H}$. We will find that the reheating temperature needs to be larger than about $1 \mathrm{GeV}$ for neutralinos to be good dark matter candidates. ${ }^{2}$

The plan of the paper is as follows. In Sec. II we briefly recall the calculation of a WIMP relic abundance both in the low-reheating scenario and in the standard case. In Sec. III the specific supersymmetric models that will be considered in our analysis are introduced. In Sec. IV we discuss our results. Section V is devoted to our conclusions.

\section{NEUTRALINO RELIC ABUNDANCE: THE STANDARD SCENARIO AND THE LOW-REHEATING-TEMPERATURE SCENARIO}

In this section we outline the ingredients that are relevant to the calculation of the neutralino relic abundance both in the standard radiation-dominated scenario and in the lowreheating early universe scenario introduced in Ref. [14]. We direct the reader to Refs. $[20,14]$ for further details.

\section{A. The standard scenario}

The number density $n_{\chi}$ of neutralinos in the early universe is governed by the Boltzmann equation which takes

\footnotetext{
${ }^{1}$ Low-reheating scenarios lead as well to a new perspective on baryogenesis [15] and to the possibility that massive neutrinos may play the role of warm dark matter [16].

${ }^{2}$ In this paper we suppose that neutralinos are produced during the reheating process only through thermal scatterings. Another source might be the direct decay of the inflaton field into neutralinos $[18,19]$. This introduces another unknown parameter though, the decay rate of the inflaton field into neutralinos, and we do not consider this possibility any further.
}

into account both the expansion of the universe and the neutralino interactions in the primordial plasma:

$$
\frac{d n_{\chi}}{d t}=-3 H n_{\chi}-\left\langle\sigma_{\mathrm{ann}} v\right\rangle\left[n_{\chi}^{2}-\left(n_{\chi}^{\mathrm{eq}}\right)^{2}\right]
$$

where $H$ is the Hubble parameter, $t$ denotes time, and $n_{\chi}^{\text {eq }}$ is the neutralino equilibrium number density. In the right-hand side of Eq. (3), the first term describes the universe expansion, while the second term takes into account the change in the $\chi$ number density due to annihilation and inverseannihilation processes.

At high temperatures, the evolution of $n_{\chi}$ closely tracks its equilibrium value $n_{\chi}^{\mathrm{eq}}$. In this regime the interaction rate of the $\chi$ particles is strong enough to keep them in thermal equilibrium with the plasma. As the temperature decreases, for heavy particles like neutralinos the quantity $n_{\chi}^{\mathrm{eq}}$ becomes exponentially suppressed and therefore the interaction rate $\Gamma=n_{\chi}\left\langle\sigma_{\mathrm{ann}} v\right\rangle$ turns out to be rapidly ineffective in maintaining neutralinos in thermal equilibrium: when the $\chi$ 's mean free path becomes of the order of the Hubble scale, $\chi$ interactions are frozen out and the $\chi$ number density per comoving volume is frozen in. This situation occurs at a temperature $T_{F}$ (freeze-out temperature) and clearly depends on the strength of the $\chi$ interactions.

By integrating the Boltzmann equation of Eq. (3) up to the present time, one finds the neutralino relic density $\Omega_{\chi} h^{2}$ :

$$
\Omega_{\chi} h^{2}=\frac{m_{\chi} n_{\chi}(T=0)}{\rho_{c}} h^{2} .
$$

A simple analytic approximation of the solution of the Boltzmann equation (3) allows us to write down explicitly both the neutralino relic abundance

$$
\Omega_{\chi} h^{2} \simeq 8.77 \times 10^{-11} \frac{1}{g_{*}^{1 / 2}\left(T_{F, \mathrm{std}}\right)} \frac{\mathrm{GeV}^{-2}}{\left\langle\sigma_{\mathrm{ann}} v\right\rangle_{\mathrm{int}}}
$$

and the value of the freeze-out temperature for the standard scenario $T_{F}=T_{F, \text { std }}$ in the implicit form

$$
x_{F, \mathrm{std}} \simeq \ln \left[0.038 \frac{g m_{\chi} M_{P} x_{F, \mathrm{std}}^{1 / 2}}{g_{*}^{1 / 2}\left(T_{F, \mathrm{std}}\right)}\left\langle\sigma_{\mathrm{ann}} v\right\rangle_{F}\right],
$$

where $x_{F, \text { std }} \equiv m_{\chi} / T_{F \text {,std }}$ and $\left\langle\sigma_{\mathrm{ann}} v\right\rangle_{F}$ denotes the value of $\left\langle\sigma_{\mathrm{ann}} v\right\rangle$ at the freeze-out temperature. In Eqs. (5),(6) $M_{P}$ denotes the Planck mass, $g$ is the number of internal degrees of freedom of $\chi, g_{*}\left(T_{F}\right)$ is the effective number of degrees of freedom of the plasma at the freeze-out, and $\left\langle\sigma_{\mathrm{ann}} v\right\rangle_{\mathrm{int}}$ denotes the integrated value of $\left\langle\sigma_{\mathrm{ann}} v\right\rangle$ from $T_{F}$ up to the present temperature. Making use of the nonrelativistic firstorder expansion of $\left\langle\sigma_{\mathrm{ann}} v\right\rangle$ in terms of the variable $x$ $\equiv m_{\chi} / T$,

$$
\left\langle\sigma_{\mathrm{ann}} v\right\rangle=\tilde{a}+\widetilde{b} x^{-1},
$$


we have $\left\langle\sigma_{\mathrm{ann}} v\right\rangle_{\mathrm{int}}=\tilde{a} x_{F}^{-1}+\tilde{b} x_{F}^{-2} / 2$. Equation (5) shows the well known result that the present abundance of a cold relic particle is inversely proportional to its annihilation cross section: $\Omega_{\chi} h^{2} \sim\left\langle\sigma_{\text {ann }} v\right\rangle_{\text {int }}^{-1}$.

A crucial point in this discussion is that, in the standard cosmological scenario, freeze-out occurs in a phase of the evolution of the universe when the expansion is adiabatic and the energy density is dominated by radiation: $T \sim a^{-1}$ and $H \sim T^{2} \sim a^{-2}$. These relations between the temperature, the scale factor $a$, and the Hubble parameter are modified in the reheating phase of the low-reheating-temperature scenario discussed in the next section: If freeze-out occurs during the reheating phase, a lower neutralino relic abundance at the present time is obtained.

\section{B. A low-reheating-temperature scenario}

It is by now accepted that during the early epochs of the universe there was a primordial stage of inflation [17] responsible for the observed homogeneity and isotropy of the present universe as well as for the generation of the cosmological perturbations.

The radiation-dominated era of the universe is usually assumed to be originated by the decay of the coherent oscillations of a scalar field, the inflaton field, whose vacuum energy drove inflation. ${ }^{3}$ The decay of the scalar field into light degrees of freedom and their subsequent thermalization, called reheating, leaves the universe at a temperature $T_{R H}$, which represents the largest temperature of the plasma during the subsequent radiation-dominated epoch, when temperature is a decreasing function of time. The onset of the radiation-dominated era is in fact placed at the temperature $T_{R H}$, i.e., at the end of the reheating phase.

Usually $T_{R H}$ is assumed to be very large and-in any case-larger than the neutralino freeze-out temperature $T_{F}$. This fact implies that the present-day relic abundance of any particle which freezes out at a temperature $T_{F}<T_{R H}$ is not affected by the history of the universe during the reheating phase. However, the only information we have on the smallest value of $T_{R H}$ is from requiring a successful period of primordial nucleosynthesis, $T_{R H} \gtrsim 1 \mathrm{MeV}$. Therefore, from a phenomenological point of view, $T_{R H}$ is actually a free parameter. This implies that the situation in which a relic particle decoupled from the plasma before reheating was completed (i.e., $T_{F}>T_{R H}$ ) could be a viable possibility, with important implications in the calculation of the cosmological abundance of relic particles [14,21].

\footnotetext{
${ }^{3}$ Identifying this scalar field with the inflaton field is not strictly necessary. It might well be identified with some massive nearly stable particle, such as some light modulus field present in supersymmetric and (super)string models. In such a case, after inflation the universe might have been matter dominated by the energy density of this modulus and then become radiation dominated after its decay. In other words, there might have been more than one reheating process during the thermal history of the universe. Needless to say, the one relevant for us is the latest.
}

Let us consider the scenario of the early universe discussed in Ref. [14]. During the reheating epoch, the energy density of the universe is dominated by the coherent oscillations of a scalar field $\phi$. This period begins at a time $H_{I}^{-1}$ and lasts until a time $\Gamma_{\phi}^{-1}$ set by the scalar field decay rate $\Gamma_{\phi}$. The dynamics of the system for $H_{I}^{-1}<t<\Gamma_{\phi}^{-1}$ is described by the Boltzmann equations for the energy densities $\rho_{\phi, R, \chi}$ of the three coupled components, the (unstable) massive field $\phi$, the radiation $R$, and the (stable) massive WIMP $\chi$ 's:

$$
\begin{aligned}
& \frac{d \rho_{\phi}}{d t}=-3 H \rho_{\phi}-\Gamma_{\phi} \rho_{\phi}, \\
& \frac{d \rho_{R}}{d t}=-4 H \rho_{R}+\Gamma_{\phi} \rho_{\phi}+\left(2\left\langle E_{\chi}\right\rangle\right)\left\langle\sigma_{\mathrm{ann}} v\right\rangle\left[n_{\chi}^{2}-\left(n_{\chi}^{\mathrm{eq}}\right)^{2}\right],
\end{aligned}
$$

$$
\frac{d n_{\chi}}{d t}=-3 H n_{\chi}-\left\langle\sigma_{\mathrm{ann}} v\right\rangle\left[n_{\chi}^{2}-\left(n_{\chi}^{\mathrm{eq}}\right)^{2}\right],
$$

where the quantity $2\left\langle E_{\chi}\right\rangle$ represents the average energy released in each $\chi \chi$ annihilation. Notice that here we assume that $\phi$ decays into radiation, but not into $\chi$ 's. This set of equations assumes that the decay products of the $\phi$ field readily thermalize, forming a thermal bath. This assumption is motivated by the fact that soft particles with energies of the order of the temperature of the thermal bath leading to full equilibrium are readily produced by inelastic scatterings which are dominated by infrared effects. LSPs may also be produced by high-energy particles produced in the cascade process induced by the decay of the $\phi$ field. However, we expect such a contribution to happen at early epochs and therefore the corresponding number density of these LSPs to be diluted by the subsequent entropy release during reheating. This additional contribution to the relic density (LSP produced by hard scatterings) can therefore be relevant only when the standard thermal production of LSPs is suppressed. This situation actually does not occur for the SUGRA scheme with neutralinos lighter than the $\mathrm{TeV}$ scale we are considering in this paper, as will be discussed later on. In the following we will recall the main properties of the system described by Eqs. (8),(9),(10), directing the reader to Ref. [14] for a complete discussion.

At very early times the energy density of the universe is dominated by the scalar field $\phi$, and both the radiation and WIMP energy densities are negligible. As the scalar field decays the temperature $T$ grows until it reaches a maximum value $T_{\max }$ and then decreases as $T \propto a^{-3 / 8}$ up to the temperature $T_{R H}$ at the time $t \simeq \Gamma_{\phi}^{-1}$ which determines the end of reheating (in this second stage the temperature of the radiation produced in the first stage is cooled down by expansion, and the entropy release due to the decay of $\phi$ induces a gentler cooling compared to the radiation-dominated case). This nonstandard relation between the temperature and the scale factor may significantly affect the calculation of the $\chi$ particles relic abundance, depending on the duration and on the details of the reheating phase. 
The relevant mass scales that can help in understanding the different regimes which may occur are the mass of the WIMP $m_{\chi}$, the temperature $T_{F}$ of the WIMP freeze-out (which can happen before or after the reheating has been completed), the temperature at the end of the reheating phase $T_{R H}$, and the maximal temperature reached during the reheating phase $T_{\max }$. Two hierarchies are present for these mass scales: $T_{R H}<T_{\max }$ and, since we are dealing with cold relics which decouple when nonrelativistic, $T_{F}<m_{\chi}$.

The relation between $m_{\chi}$ and $T_{\max }$ determines whether the WIMPs, which are produced during the reheating phase, become relativistic $\left(T_{\max }>m_{\chi}\right)$ or not $\left(T_{\max }<m_{\chi}\right)$. More important is to determine whether the $\chi$ particles reach thermal equilibrium during the reheating phase. This is determined by the strength of their interactions, and in particular it depends on $\left\langle\sigma_{\mathrm{ann}} v\right\rangle$. According to the values of the WIMP mass and annihilation cross section, two possible nonstandard regimes may be schematically singled out for the WIMP relic abundance.

(i) The $\chi$ particles generated during reheating are always nonrelativistic and never reach thermal equilibrium. Integration of Eqs. (8),(9),(10) shows that the process of $\chi$ production takes its main contribution around the temperature $T_{*}$ $\simeq m_{\chi} / 4$ and is exponentially suppressed outside a narrow interval centered on $T_{*}$ [14]. So most of the $\chi$ particles are produced at $T_{*}$. For $T<T_{*}$ the total number of $\chi$ 's is frozen and their density is diluted by expansion. The condition $n_{\chi}(T=0)<n_{\chi}^{\mathrm{eq}}\left(T_{*}\right)$ implies an upper limit on $\left\langle\sigma_{\mathrm{ann}} v\right\rangle_{*}$ $\equiv\left\langle\sigma_{\mathrm{ann}} v\right\rangle_{\left(T=T_{*}\right)}$ of the order of [14]:

$$
\begin{aligned}
\left\langle\sigma_{\mathrm{ann}} v\right\rangle_{*} \lesssim & 7 \times 10^{-14} \frac{2}{g}\left[\frac{g_{*}\left(T_{*}\right)}{10}\right]\left[\frac{10}{g_{*}\left(T_{R H}\right)}\right]^{1 / 2}\left(\frac{m_{\chi}}{100 \mathrm{GeV}}\right) \\
& \times\left(\frac{100 \mathrm{MeV}}{T_{R H}}\right)^{2} \mathrm{GeV}^{-2}
\end{aligned}
$$

In this regime the WIMP relic density turns out to be proportional to the WIMP self-annihilation cross section: $\Omega_{\chi} h^{2} \sim\left\langle\sigma_{\mathrm{ann}} v\right\rangle_{*}$, instead of being inversely proportional, as in the standard case. We anticipate here that for the case of neutralinos in supersymmetric models, this situation only occurs when the reheating temperature is smaller than about $300 \mathrm{MeV}$. In this situation the neutralino relic density is significantly suppressed.

(ii) The $\chi$ particles reach thermal equilibrium, and then freeze out when nonrelativistic before the reheating phase is concluded, i.e., at a temperature $T_{F}=T_{F, r h}>T_{R H}$. During the phase when they reach thermal equilibrium, the $\chi$ particles may or may not become relativistic, depending on the value of $T_{\max }$. In any case, they decouple as nonrelativistic, leading to a cold relic. The usual freeze-out condition is modified compared to the standard case because the energy density is dominated by the scalar field and the relation between the Hubble constant and the temperature is given by $H \propto T^{4}$, as compared to the radiation-dominated case where $H \propto T^{2}$. The first consequence of this fact is that freeze-out occurs earlier and the WIMP density at $T_{F, r h}$ turns out to be higher. However, as the universe cools down from $T_{F, r h}$ to
$T_{R H}$, due to the entropy produced by the $\phi$ decays, the expansion dilutes $n_{\chi}$ by a factor $\left(T_{R H} / T_{F, r h}\right)^{8}$, which is much smaller than the dilution factor in the radiation-dominated case $\left(T_{R H} / T_{F, \text { std }}\right)^{3}$. When the two effects are combined, the final result is a suppression of $\Omega_{\chi}$ by roughly a factor $T_{R H}^{3} T_{F, \text { std }} /\left(T_{F, r h}\right)^{4}$ as compared to the standard case. An analytic approximation of the ensuing result for the $\chi$ relic abundance is given by [14]

$$
\Omega_{\chi} h^{2} \simeq 2.3 \times 10^{-11} \frac{g_{*}^{1 / 2}\left(T_{R H}\right)}{g_{*}\left(T_{F, r h}\right)} \frac{T_{R H}^{3} \mathrm{GeV}^{-2}}{m_{\chi}^{3}\left(\tilde{a} x_{F, r h}^{-4}+4 \tilde{b} x_{F, r h}^{-5} / 5\right)},
$$

where the freeze-out temperature during the reheating phase $T_{F, r h}\left(x_{F, r h} \equiv m_{\chi} / T_{F, r h}\right)$ is [14]

$$
x_{F, r h} \simeq \ln \left[0.015 \frac{g g_{*}^{1 / 2}\left(T_{R H}\right)}{g_{*}\left(T_{F, r h}\right)} \frac{M_{P} T_{R H}^{2}}{m_{\chi}}\left(\tilde{a} x_{F, r h}^{5 / 2}+5 \tilde{b} x_{F, r h}^{3 / 2} / 4\right)\right] .
$$

In both Eqs. (12) and (13) we have used the annihilation cross section expansion of Eq. (7).

Obviously, if the decoupling of the $\chi$ particles from the plasma occurs after the reheating phase is concluded, the standard scenario is recovered and the relic abundance has the ordinary expression of Eq. (5).

In the following sections we will perform a detailed calculation of the neutralino relic abundance in a supergravity framework in order to study the consequences of the lowreheating-temperature scenario outlined above. In our analysis we will make use of the analytical solutions given in Eqs. (5),(6), and Eqs. (12),(13), appropriately interpolated in the intermediate regime where $T_{F}$ is close to $T_{R H}$ (the interpolation has been determined on the basis of numerical solutions of the relevant differential equations). We have numerically verified that the analytical solutions are accurate enough for our purposes when compared with full numerical solution of the relevant Boltzmann equations of Eq. (3) and Eqs. (8),(9),(10).

\section{THE NEUTRALINO IN MINIMAL SUPERGRAVITY}

Supersymmetric theories naturally predict the existence of viable dark matter candidates if $R$ parity is conserved, since this symmetry prevents the lightest supersymmetric particle from decaying. The nature and the properties of the LSP depend on the way supersymmetry is broken. In models where supersymmetry breaking is realized through gravity(or also anomaly-)mediated mechanisms, the LSP turns out to be quite naturally the neutralino, defined as the lowestmass linear superposition of the photino $(\tilde{\gamma}), z$-ino $(\widetilde{Z})$, and the two Higgsino states $\left(\widetilde{H}_{1}^{\circ}, \widetilde{H}_{2}^{\circ}\right): \chi \equiv a_{1} \tilde{\gamma}+a_{2} \widetilde{Z}+a_{3} \widetilde{H}_{1}^{\circ}$ $+a_{4} \widetilde{H}_{2}^{\circ}$.

Even assuming a minimal supersymmetric extension of the standard model, the supersymmetric theories may be explored in a variety of different schemes, ranging from those based on universal [3-7,9-13] or nonuniversal $[3-5,8,11,12]$ supergravity, where the relevant independent 
supersymmetric parameters are defined at a grand unification scale, to effective supersymmetric theories which are defined at the electroweak scale $[5,22,23]$. In the present paper we will mostly concentrate on the discussion of universal supergravity and we will comment on the results that can be obtained in different supersymmetric schemes.

The essential elements of a generic minimal supersymmetric model are described by a Yang-Mills Lagrangian, by the superpotential, which contains all the Yukawa interactions between the standard and supersymmetric fields, and by the soft-breaking Lagrangian, which models the breaking of supersymmetry. Implementation of this model within a supergravity scheme leads quite naturally to a set of unification conditions at a grand unification scale $\left(M_{\mathrm{GUT}}\right)$ for the parameters of the theory.

Unification of the gaugino masses:

$$
M_{i}\left(M_{\mathrm{GUT}}\right) \equiv m_{1 / 2},
$$

universality of the scalar masses with a common mass denoted by $m_{0}$ :

$$
m_{i}\left(M_{\mathrm{GUT}}\right) \equiv m_{0},
$$

and universality of the trilinear scalar couplings:

$$
A^{l}\left(M_{\mathrm{GUT}}\right)=A^{d}\left(M_{\mathrm{GUT}}\right)=A^{u}\left(M_{\mathrm{GUT}}\right) \equiv A_{0} m_{0} .
$$

We denote this scheme as universal SUGRA (or minimal SUGRA). The relevant parameters of the model at the electroweak scale are obtained from their corresponding values at the $M_{\mathrm{GUT}}$ scale by running these down according to renormalization group equations. By requiring that the electroweak symmetry breaking is induced radiatively by the soft supersymmetry breaking, one finally reduces the parameters of the model to five: $m_{1 / 2}, m_{0}, A_{0}, \tan \beta \equiv v_{2} / v_{1}$, and $\operatorname{sgn}(\mu)$, where $v_{1}$ and $v_{2}$ denote the vacuum expectation values of the two Higgs fields of the model and $\mu$ is a mixing parameter between the two Higgs fields which enters in the superpotential. These parameters are a priori undetermined. However, bounds coming from supersymmetry and Higgs boson searches at accelerators and on supersymmetric contributions to rare processes, like the $b \rightarrow s+\gamma$ radiative decay, introduce limits on the model parameter space. Also theoretical arguments concerning the naturalness of the theory may be used in order to identify typical scales beyond which the main attractive features of supersymmetry fade away. For instance, fine-tuning arguments may be invoked to set bounds on $m_{0}$ and $m_{1 / 2}[3,4]: m_{1 / 2} \leqslant$ hundreds of $\mathrm{GeV}$, whereas $m_{0} \lesssim 2-3 \mathrm{TeV}$.

In the present paper we will vary the parameters of the minimal SUGRA scheme in wide ranges in order to carefully analyze the impact of the low-reheating-temperature cosmology on relic neutralinos. The ranges we adopt are the following:

$$
\begin{gathered}
50 \mathrm{GeV} \leqslant m_{1 / 2} \leqslant 3 \mathrm{TeV}, \\
m_{0} \leqslant 3 \mathrm{TeV}, \\
-3 \leqslant A_{0} \leqslant+3, \\
1 \leqslant \tan \beta \leqslant 60 .
\end{gathered}
$$

The sign of $\mu$ is chosen to be positive, since negative values are somewhat disfavored by the limits on the supersymmetric contribution to the muon anomalous magnetic moment. On the configurations obtained by randomly scanning the above defined parameter space, we apply the experimental limits quoted above on Higgs boson and supersymmetry searches and on the $b \rightarrow s+\gamma$ decay (for details, see, for instance, Refs. [24,25]).

We finally remark that the phenomenology of relic neutralinos, in some sectors of the minimal SUGRA scheme, is also quite sensitive to some standard model parameters, such as the top quark mass $m_{t}$, the bottom quark mass $m_{b}$, and the strong coupling $\alpha_{s}[6,25,26]$. For these parameters, we use here their 95\% C.L. ranges: $m_{t}^{\text {pole }}=175 \pm 10 \mathrm{GeV}$, $m_{b}\left(M_{Z}\right)=3.02 \pm 0.21 \mathrm{GeV}$, and $\alpha_{s}\left(M_{Z}\right)=0.118 \pm 0.004$.

\section{RESULTS AND DISCUSSION}

As already discussed in Sec. II, the calculation of the neutralino relic abundance $\Omega_{\chi} h^{2}$, both in the standard case outlined in Sec. II A and in the low-reheating scenario described in Sec. II B, relies on a detailed calculation of the neutralino self-annihilation cross section $\left\langle\sigma_{\mathrm{ann}} v\right\rangle$. Here we consider the universal SUGRA model outlined in the previous section, and calculate $\left\langle\sigma_{\mathrm{ann}} v\right\rangle$ following the procedure given in Ref. [20], to which we refer for details.

A first preliminary conclusion about neutralino dark matter and models with a low reheating temperature may be drawn by calculating the neutralino annihilation cross section at the temperature $T_{*}$. The quantity $\left\langle\sigma_{\text {ann }} v\right\rangle_{*}$ is shown in Fig. 1 as a function of $m_{\chi}$ in terms of a scatter plot obtained by varying the SUGRA parameters in the ranges given by Eq. (17). The scatter plot is compared to the values of the limiting cross section given in Eq. (11), calculated for different values of $T_{R H}$. This figure shows that the condition of nonrelativistic nonequilibrium, given by Eq. (11), is verified only for values of $T_{R H}$ smaller than about $300 \mathrm{MeV}$. For larger values of the reheating temperature, $\left\langle\sigma_{\mathrm{ann}} v\right\rangle_{*}$ always lies above the curves of the limiting cross section. This fact implies that for $T_{R H} \gtrsim 300 \mathrm{MeV}$ the neutralino always reaches thermal equilibrium during the reheating phase, and therefore the peculiar behavior $\Omega_{\chi} h^{2} \propto\left\langle\sigma_{\text {ann }} v\right\rangle$ is limited only to cosmological models with very low reheating temperatures. This conclusion is actually true for a class of supersymetric scenarios which is more general than the universal SUGRA model shown in Fig. 1. For instance, we have explicitly verified that the same result also applies to other SUGRA schemes where the universality condition of Eq. (15) are relaxed at the GUT scale for the Higgs sector, when the supersymmetric parameters are varied in the same ranges discussed in the previous section. One must notice that the 


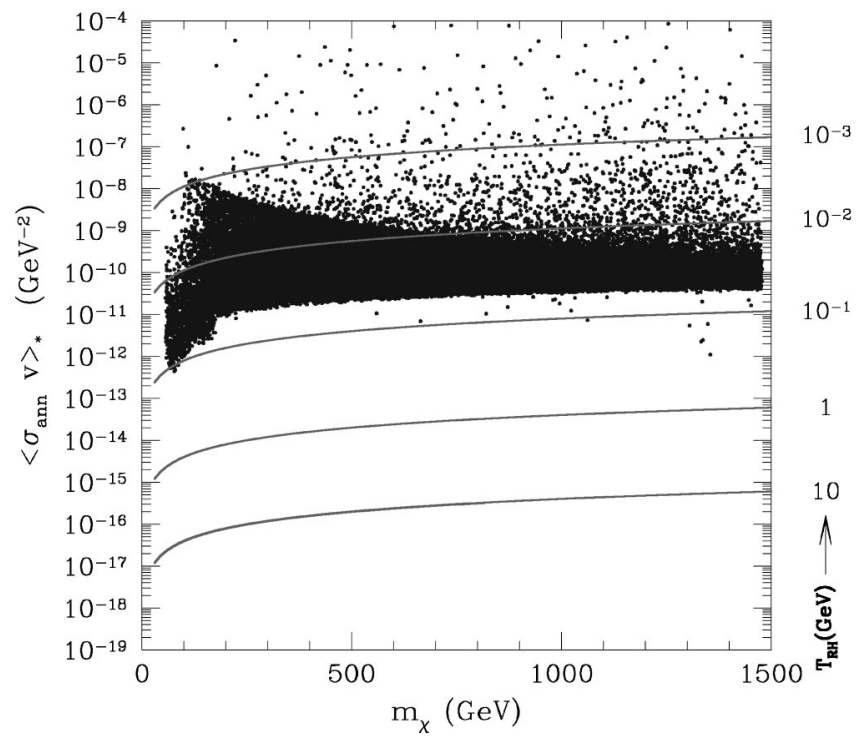

FIG. 1. Thermal average, at the temperature $T_{*} \sim m_{\chi} / 4$, of the neutralino self-annihilation cross section times the relative velocity $\left\langle\sigma_{\mathrm{ann}} v\right\rangle_{*}$ as a function of the neutralino mass $m_{\chi}$. The points denote the values of $\left\langle\sigma_{\mathrm{ann}} v\right\rangle_{*}$ calculated in universal SUGRA with the parameters varied as in Eq. (17). The values of $m_{t}^{\text {pole }}, m_{b}$, and $\alpha_{s}$ are varied inside their $2 \sigma$ allowed intervals. The solid lines denote, for different values of the reheating temperature $T_{R H}$, the values of the limiting cross section of Eq. (11) which determines, for a cosmological model where neutralinos are always nonrelativistic, whether neutralinos can reach thermal equilibrium during the reheating phase.

effective lower limit on neutralino cross section revealed in Fig. 1 is a consequence of the choice of the upper ranges of Eq. (17) adopted for the dimensional supersymmetric parameters. However, we recall that the intervals given in Eq. (17) are representative of a typical upper bound on the supersymmetry breaking scale, $M_{\text {SUSY }} \lesssim$ a few $\times 10^{3} \mathrm{GeV}$. This constraint may be understood on quite general grounds, since it derives from naturalness arguments on the stability of the Higgs potential and the requirement of absence of finetuning in the generation of the electroweak scale through the mechanism of radiative symmetry breaking. Notice that, as far as $m_{1 / 2}$ is concerned, the upper value adopted in Eq. (17) is already large enough to pose questions about fine-tuning problems $[3,4]$.

Let us turn now to the calculation of the neutralino relic abundance. Figure 2 shows $\Omega_{\chi} h^{2}$ as a function of the neutralino mass $m_{\chi}$, calculated in universal SUGRA for $\tan \beta$ $=30$ and $A_{0}=0$ and with $m_{0}$ and $m_{1 / 2}$ varied in the ranges of Eq. (17). Starting from the upper left panel, we plot the results obtained for standard cosmology, and for lowreheating-temperature cosmologies with $T_{R H}=30,20,10,5$, $1 \mathrm{GeV}$. In the case of standard cosmology, the neutralino relic abundance turns out to be generically quite large, in excess of the upper bound on the total amount of matter in the universe given in Eq. (2). This is a typical feature of universal SUGRA models for values of $\tan \beta \leqq 40$ [3-7,913]. For these schemes, the constraint on the parameter space coming from cosmology is actually very strong, especially in posing stringent upper limits on the neutralino mass. Figure 2 shows that an upper bound of about $200 \mathrm{GeV}$ is obtained on the neutralino mass when we restrict $\Omega_{\chi} h^{2}$ to be less than 0.3 .

There are, however, ways out to avoid such a bound. When coannihilation is included, the upper bound on $m_{\chi}$ in universal SUGRA at low/intermediate $\tan \beta$ can be extended up to about $500 \mathrm{GeV}[6,7,11,13]$, even though this possibility is restricted to a very narrow sector of the SUGRA parameter space where the neutralino mass is almost degenerate with the stau mass.

Alternatively, when a low reheating temperature is allowed, Fig. 2 shows that the upper limit on $m_{\chi}$ coming from cosmology is removed. By lowering the reheating temperature we affect mainly the relic abundance for large neutralino masses, unless we lower $T_{R H}$ below a few GeV: for $T_{R H}$ $\$ 5 \mathrm{GeV}, \Omega_{\chi} h^{2}$ is reduced for all the allowed mass range of the SUGRA model.

The behavior of the different panels of Fig. 2 may be easily understood by comparing the relevant mass scales which enter in the calculation of the relic abundance in the standard and low-reheating-temperature models, namely, $m_{\chi}, T_{F}$, and $T_{R H}$. In Fig. 3 we show a generic example of what happens at different neutralino masses for a sufficiently low reheating temperature. The upper panel of Fig. 3 shows the relic abundance vs $m_{\chi}$, calculated for $\left\langle\sigma_{\mathrm{ann}} v\right\rangle$ fixed at the value $10^{-10} \mathrm{GeV}^{-2}$. The thick horizontal line refers to the calculation for standard cosmology, while the thick decreasing line refers to $T_{R H}=10 \mathrm{GeV}$. The two lines are superimposed for $m_{\chi} \lesssim 150 \mathrm{GeV}$. The lower panel shows the value of the freeze-out temperature $T_{F}$ as a function of the neutralino mass. The horizontal line indicates the values of $T_{R H}=10 \mathrm{GeV}$. By comparing the upper an lower panels, we see that as long as the freeze-out occurs at a temperature which is (much) smaller than $T_{R H}$, no difference is present for the low-reheating-temperature scenario as compared to the standard case. On the contrary, when $T_{F}$ approaches $T_{R H}$, the relic abundance becomes suppressed, and this effect becomes more pronounced as $T_{F}$ grows as compared to $T_{R H}$. Since $T_{F}$ is an increasing function of the neutralino mass, a low-reheating scenario affects more the large mass sector of the theory, and this explains the peculiar behavior of Fig. 2.

Going back to the discussion of the features of Fig. 2, we see that, in order to have heavy neutralinos compatible with the cosmological upper bound in Eq. (2), the reheating temperature has to be lowered below about $30 \mathrm{GeV}$. When $T_{R H}$ falls below $20 \mathrm{GeV}$ the whole neutralino mass range is acceptable from the point of view of cosmology, although a fraction of the SUGRA configurations entail relic neutralinos which are a subdominant dark matter component, since $\Omega_{\chi} h^{2}$ falls below the lower limit of Eq. (2). When $T_{R H}$ $\$ 0.6 \mathrm{GeV}$ all the SUGRA configurations lead to subdominant relic neutralinos. For smaller values of $T_{R H}$ the neutralino relic abundance is even more suppressed and becomes negligible for $T_{R H}$ in the $\mathrm{MeV}$ range.

We comment at this point that for larger values of $\tan \beta(\tan \beta \geq 40)$ in SUGRA models an upper limit on the neutralino mass is not present even in standard cosmology. 

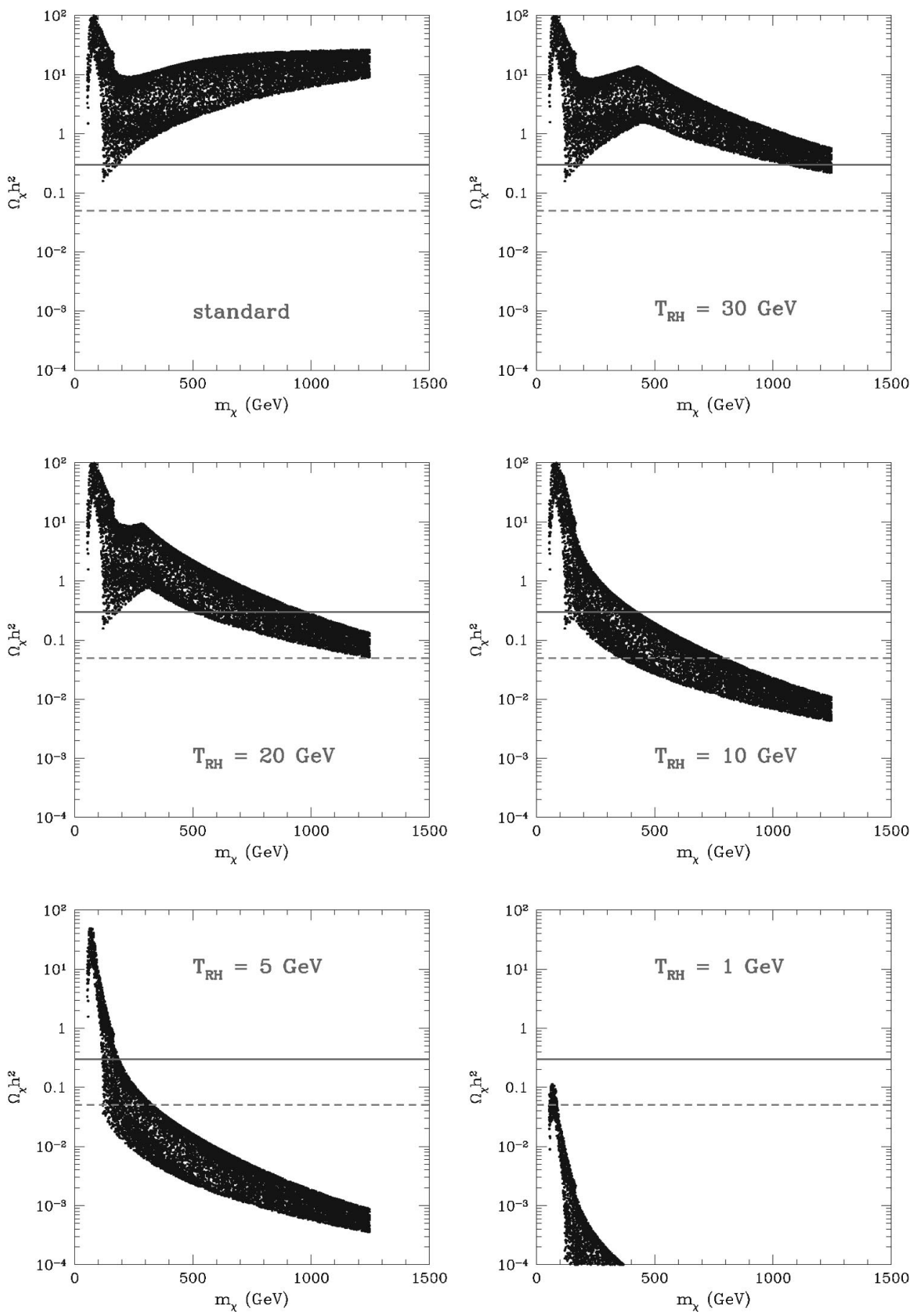

FIG. 2. Neutralino relic density $\Omega_{\chi} h^{2}$ as a function of the neutralino mass $m_{\chi}$ in universal SUGRA, for $\tan \beta=30$ and $A_{0}=0$. The parameters $m_{0}$ and $m_{1 / 2}$ are varied according to the intervals of Eq. (17). The values of $m_{t}^{\text {pole }}, m_{b}$, and $\alpha_{s}$ are fixed at their central values: $m_{t}^{\text {pole }}=175 \mathrm{GeV}, m_{b}\left(M_{Z}\right)=3.02 \mathrm{GeV}$, and $\alpha_{s}\left(M_{Z}\right)=0.118$. The upper left panel shows results for standard cosmology. The other panels refer to different values of the reheating temperature $T_{R H}$. The horizontal solid and dashed lines delimit the interval for the amount of nonbaryonic dark matter in the universe, given in Eq. (2).

The occurrence of an acceptable relic abundance is generically confined to corridors in the parameter space where either the relic abundance is suppressed by coannihilation or the neutralino mass lies close to the pole of the annihilation cross section mediated by the pseudoscalar Higgs field $A$. In a low-reheating-temperature cosmology, these features are relaxed and conclusions similar to those discussed above in connection with Fig. 2 are present. In particular, a scale of about $1 \mathrm{GeV}$ as a lower limit on $T_{R H}$ in order to have dominant relic neutralinos is present for all the values of $\tan \beta$ given in Eq. (17).

New features in the parameter space are also present when the universality is not extended to Higgs boson masses $[3,4,8,11]$. The effects of nonuniversality modify the low- 

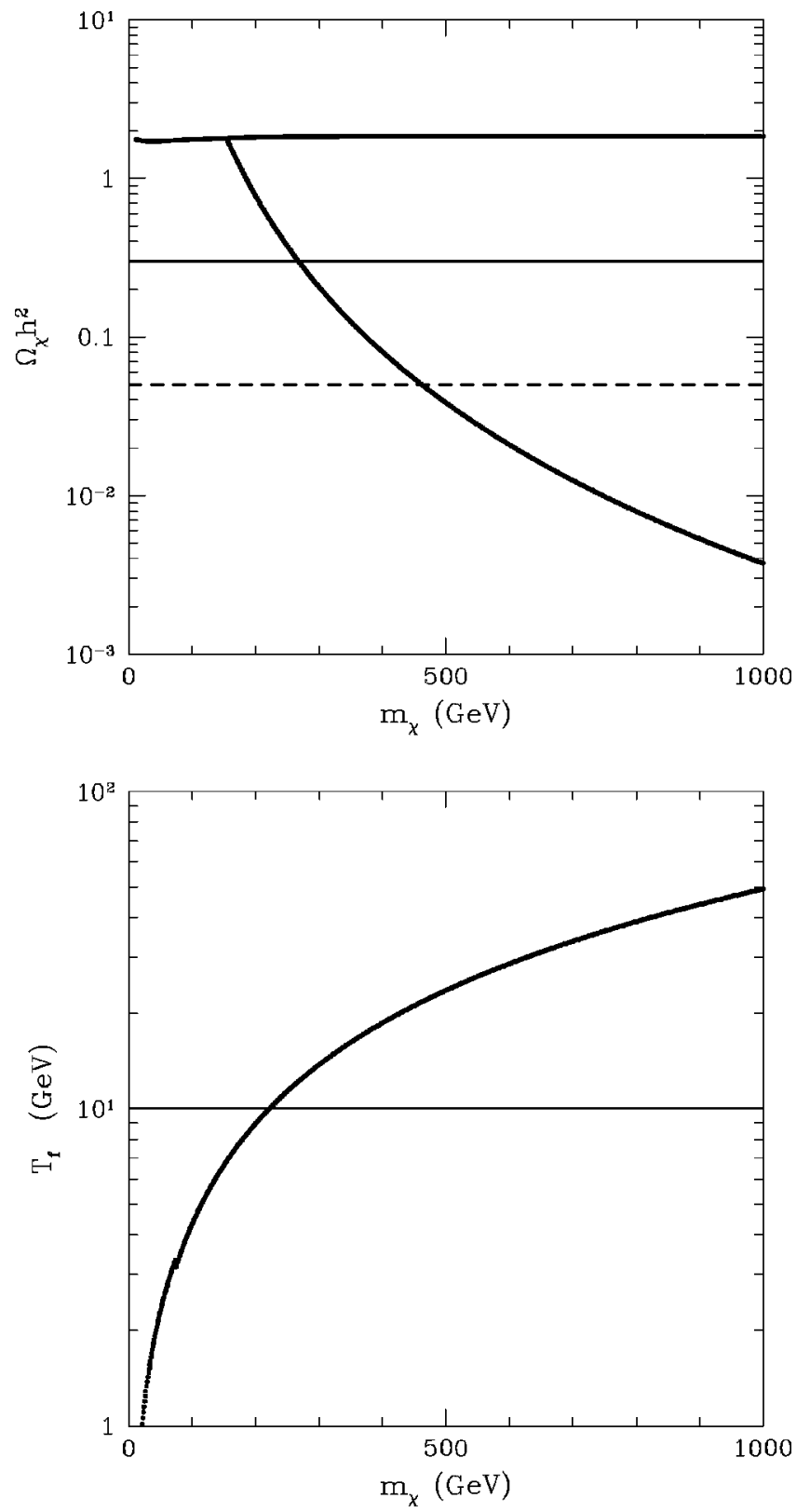

FIG. 3. Effect of a low reheating temperature on the WIMP relic abundance. The upper panel shows $\Omega_{\chi} h^{2}$ as a function of $m_{\chi}$ for standard cosmology (upper thick horizontal line) and for $T_{R H}$ $=10 \mathrm{GeV}$ (thick decreasing line). The two lines are superimposed for $m_{\chi} \lesssim 150 \mathrm{GeV}$. The thin horizontal solid and dashed lines delimit the interval for the amount of nonbaryonic dark matter in the universe, given in Eq. (2). The lower panel shows the freeze-out temperature $T_{F}$ as a function of $m_{\chi}$. The horizontal line denotes the values of the reheating temperature: $T_{R H}=10 \mathrm{GeV}$.

energy sector of the theory, through the renormalization group equation evolution and the conditions of radiative electroweak symmetry breaking: this alters the neutralino couplings and the mass spectrum of sparticles and induces variations of the neutralino annihilation cross section. The consequence is a change and an extension of the regions of parameter space that are compatible with a relic neutralino, also in standard cosmology. A low reheating temperature has again the effect of enlarging these cosmologically relevant sectors of the parameter space. A lower limit of about $1 \mathrm{GeV}$ on $T_{R H}$ in order to have dominant relic neutralinos is again recovered also in nonuniversal SUGRA models.

Figure 4 shows the same information contained in Fig. 2, expressed in the plane $m_{0}-m_{1 / 2}$. The upper left panel refers to standard cosmology; the other panels refer to lowreheating-temperature cosmologies with $T_{R H}=30,20,10,5$, $1 \mathrm{GeV}$. The value of $\tan \beta$ is fixed at 30 and $A_{0}=0$. The light-shaded regions are excluded domains: the ones on the left side of the $m_{0}-m_{1 / 2}$ plane are excluded by the experimental bounds discussed in the previous section or by the nonoccurrence of radiative electroweak symmetry breaking, while the ones on the lower part of the plane do not correspond to viable LSP neutralino models. The dark-shaded areas correspond to the domains where the neutralino relic abundance falls inside the cosmologically relevant range of Eq. (2). The hatched regions correspond to neutralinos with a subdominant relic abundance, i.e., to $\Omega_{\chi} h^{2}<0.05$.

The first panel shows that for standard cosmology the region of the $m_{0}-m_{1 / 2}$ parameter space which is allowed by cosmology is quite restricted, as discussed above. The upper limit on the acceptable values of the neutralino relic abundance imposes severe bounds on both $m_{0}$ and $m_{1 / 2}$, of the order of a few hundreds of $\mathrm{GeV}$. When coannihilation is included [6,7,11-13], the allowed region is extended in a thin band close to the lower excluded area. However, as is evident from the other panels, when the reheating temperature is lowered the consequent suppression of $\Omega_{\chi} h^{2}$ weakens considerably the constraints on $m_{0}$ and $m_{1 / 2}$. In particular, the regions of the SUGRA parameter space that are compatible with the assumption of a dominant neutralino dark matter change significantly, depending on the actual value of $T_{R H}$. For values of $T_{R H}$ below about $20 \mathrm{GeV}$ a large fraction of the parameter space is allowed by cosmology and for $T_{R H} \lesssim 1 \mathrm{GeV}$ the whole supersymmetric parameter space is compatible with the cosmological abundance of relic neutralinos, even though for most of the values of the parameters the relic abundance falls below the interval of Eq. (2).

As we discussed before, the extension of the cosmologically allowed regions in the plane $m_{0}-m_{1 / 2}$ is enlarged, in standard cosmology, when $\tan \beta$ is larger than about 40, since in this case coannihilation or annihilation through the $A$ pole is effective in reducing the values of $\Omega_{\chi} h^{2}$. In the latter case, an almost diagonal allowed band opens up $[6,26]$. Larger regions are also allowed in nonuniversal SUGRA models. In both cases, a low reheating temperature has again the effect of widely enlarging the cosmologically relevant domains in the plane $m_{0}-m_{1 / 2}$. We obtain, for the SUGRA models with $\tan \beta \geq 40$ and for the nonuniversal SUGRA schemes, that in all the supersymmetric parameter space the neutralino relic abundance is compatible with the range of Eq. (2) when $T_{R H} \lesssim 1 \mathrm{GeV}$.

In the above discussion, we noticed that, for any given neutralino mass, low enough values of $T_{R H}$ would imply a suppression of $\Omega_{\chi} h^{2}$ too strong to be compatible with the hypothesis of dominant neutralino dark matter. We can therefore use this argument to obtain a lower limit on $T_{R H}$ under the assumption that the neutralino represents the dominant 

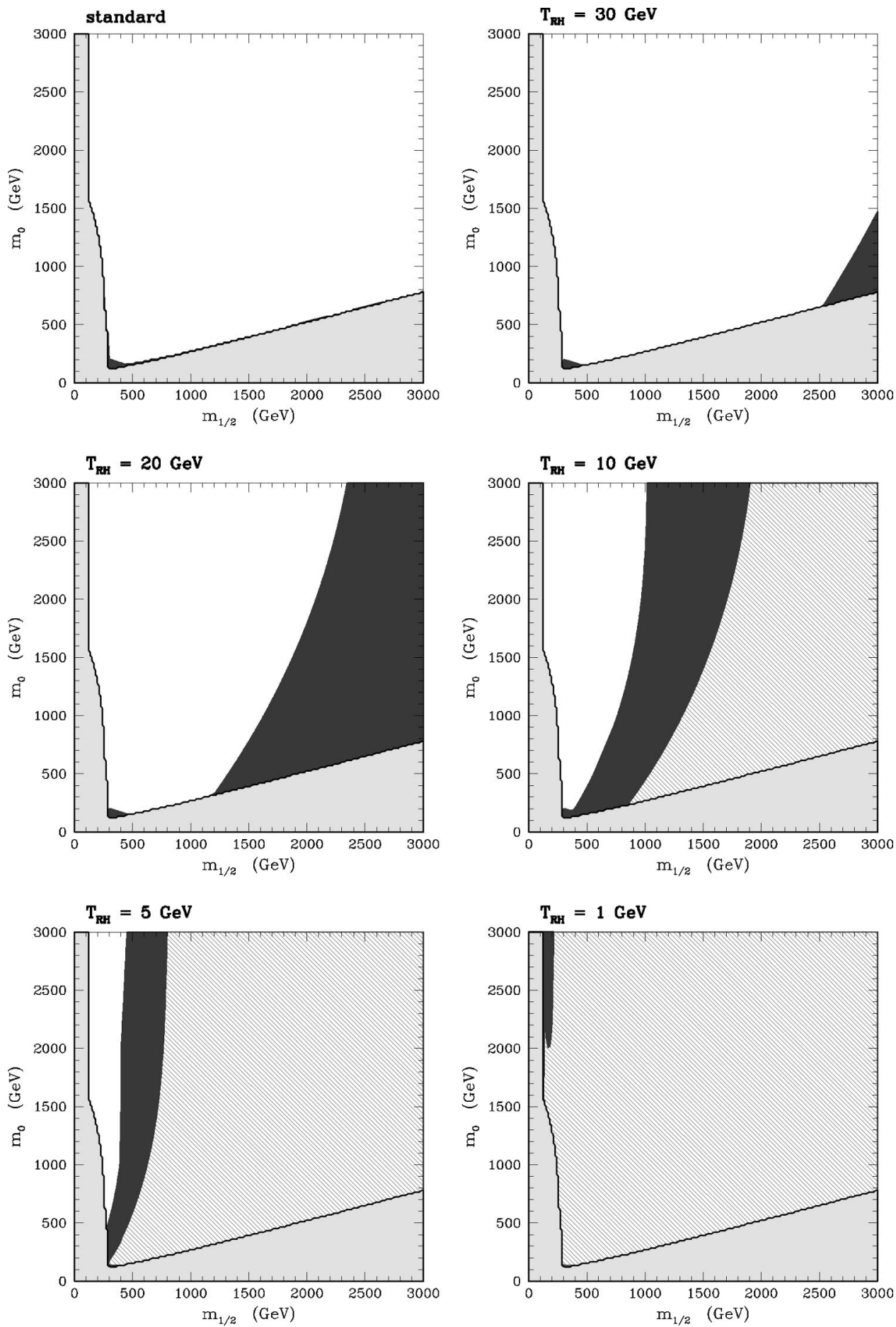

FIG. 4. Cosmologically favored regions in the plane $m_{1 / 2}$ $-m_{0}$ in universal SUGRA for $\tan \beta=30$ and $A_{0}=0$. The values of $m_{t}^{\text {pole }}, m_{b}$, and $\alpha_{s}$ are fixed at their central values: $m_{t}^{\text {pole }}=175 \mathrm{GeV}, m_{b}\left(M_{Z}\right)$ $=3.02 \mathrm{GeV}, \quad$ and $\quad \alpha_{s}\left(M_{Z}\right)$ $=0.118$. The upper left panel shows results for standard cosmology. The other panels refer to different values of the reheating temperature $T_{R H}$. The dark-shaded areas correspond to configurations where $0.05 \leqslant \Omega_{\chi} h^{2} \leqslant 0.3$. Hatched areas denote configurations where $\Omega_{\chi} h^{2}<0.05$. Light-shaded regions are excluded either by experimental constraints or by theoretical arguments. component of dark matter in the universe. This lower bound on $T_{R H}$ is shown as a solid line in Fig. 5, as a function of $m_{\chi}$. To derive this limit we have varied all the SUGRA parameters in the intervals of Eq. (17). The result plotted in Fig. 5 shows that, if we require the neutralino to be the dominant dark matter component, the reheating temperature in the early universe cannot be lower than a value ranging from $0.6 \mathrm{GeV}$ up to about $20 \mathrm{GeV}$, depending on the value of the neutralino mass. This bound on $T_{R H}$ at the GeV scale is quite interesting, since it is sizably stronger than the constraint given by nucleosynthesis. We have verified that the same result remains valid also for nonuniversal SUGRA models. Therefore a lower limit of about $1 \mathrm{GeV}$ on $T_{R H}$, in order to explain the dark matter content of the universe in terms of relic neutralinos, is a specific feature of supergravity models.

\section{CONCLUSIONS}

In standard cosmology it is usually assumed that the temperature $T_{R H}$ of the universe at the beginning of the radiation-dominated era is much higher than the supersym- 


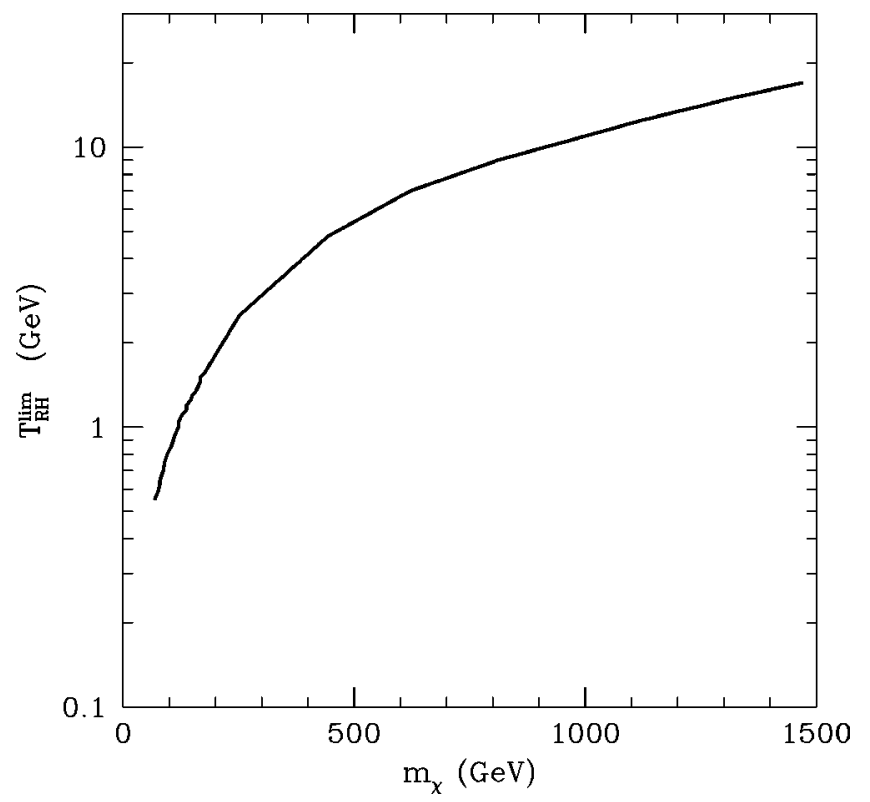

FIG. 5. Lower limit on the reheating temperature $T_{R H}$ as a function of the neutralino mass $m_{\chi}$, obtained by requiring that the neutralino is the dominant component of dark matter in the universe (i.e., $0.05 \leqslant \Omega_{\chi} h^{2} \leqslant 0.3$ ). The result refers to universal SUGRA with the parameters varied as in Eq. (17). The values of $m_{t}^{\text {pole }}, m_{b}$, and $\alpha_{s}$ are varied inside their $2 \sigma$ allowed intervals.

metry breaking scale. Moreover, neutralinos decouple from the thermal bath after the reheating phase, which followed the end of inflation, has terminated. Under these assumptions, the allowed parameter space of SUGRA models turns out to be severely constrained by the requirement that the neutralino relic density does not exceed the maximal value of the matter density of the universe deduced from observations.
In this paper we performed a quantitative study of the neutralino relic abundance in cosmological scenarios with a low reheating temperature [14]. This is a viable possibility since the only robust lower bound on the reheating temperature $T_{R H}$ can be set at the $\mathrm{MeV}$ scale, in order not to spoil nucleosynthesis predictions. The suppression on $\Omega_{\chi} h^{2}$ is originated by the fact that neutralinos decouple from the thermal bath before the end of the reheating phase. In this case the neutralino number density is diluted by entropy production and by a higher expansion rate than in the radiationdominated era.

For values of $T_{R H} \lesssim 30 \mathrm{GeV}$ the domains of the SUGRA parameter space that are compatible with dominant relic neutralinos are greatly enhanced with respect to the standard cosmological case. These domains depend on $T_{R H}$ and we have shown their evolution as a function of the reheating temperature. For $T_{R H} \lesssim 1 \mathrm{GeV}$ the whole SUGRA parameter space becomes compatible with the bounds on the dark matter relic abundance (even though the neutralino relic abundance for these low values of $T_{R H}$ is strongly suppressed).

Since lower $T_{R H}$ imply smaller relic densities, the assumption that neutralinos provide a major contribution to the dark matter of the universe implies a lower limit on $T_{R H}$. This constraint ranges from $0.6 \mathrm{GeV}$ for neutralino masses of the order of a few tens of $\mathrm{GeV}$, up to $20 \mathrm{GeV}$ for neutralino masses in the TeV range. This bound on $T_{R H}$, subject to the request of explaining the dark matter content of the universe only in terms of relic neutralinos in SUGRA schemes, is much stronger than the limit on $T_{R H}$ coming from nucleosynthesis. Similar conclusions occur also for nonuniversal SUGRA models.

\section{ACKNOWLEDGMENT}

We wish to thank G. F. Giudice for useful discussions.
[1] For recent reviews on the subject, see, for instance, Proceedings of "Topics in Astroparticle and Underground Physics" (TAUP 2001), Gran Sasso, Italy, 2001 [Nucl. Phys. B (Proc. Suppl.) B110 (2002)].

[2] W. L. Freedman, Int. J. Mod. Phys. A 17S1, 58 (2002); C. H. Lineweaver, in Proceedings of COSMO-01, Rovaniemi, Finland, 2001, astro-ph/0112381; J. R. Primack, in Proceedings of "Sources and Detection of Dark Matter and Dark Energy in the Universe (DM2002)," Marina del Rey, California, 2002, astro-ph/0205391.

[3] V. Berezinsky, A. Bottino, J. R. Ellis, N. Fornengo, G. Mignola, and S. Scopel, Astropart. Phys. 5, 1 (1996).

[4] V. Berezinsky, A. Bottino, J. R. Ellis, N. Fornengo, G. Mignola, and S. Scopel, Astropart. Phys. 5, 333 (1996).

[5] A. Bottino, F. Donato, N. Fornengo, and S. Scopel, Phys. Rev. D 59, 095004 (1999).

[6] J. Ellis, S. Heinemeyer, K. A. Olive, and G. Weiglein, Phys. Lett. B 515, 348 (2001).

[7] J. R. Ellis, T. Falk, G. Ganis, and K. A. Olive, Phys. Rev. D 62, 075010 (2000).
[8] J. R. Ellis, K. A. Olive, and Y. Santoso, Phys. Lett. B 539, 107 (2002).

[9] J. Ellis, T. Falk, G. Ganis, K. A. Olive, and M. Srednicki, Phys. Lett. B 510, 236 (2001); J. Ellis and K. A. Olive, ibid. 514, 114 (2001); J. Ellis, A. Ferstl, and K. A. Olive, Phys. Rev. D 63, 065016 (2001).

[10] J. L. Feng, K. Matchev, and F. Wilczek, Phys. Lett. B 482, 388 (2000); J. L. Feng, K. Matchev, and T. Moroi, hep-ph/0003138; J. L. Feng and K. Matchev, Phys. Rev. D 63, 095003 (2001).

[11] E. Accomando, R. Arnowitt, B. Dutta, and Y. Santoso, Nucl. Phys. B585, 124 (2000); R. Arnowitt, B. Dutta, and Y. Santoso, hep-ph/0008336.

[12] A. Corsetti and P. Nath, Phys. Rev. D 64, 125010 (2001); U. Chattopadhyay, A. Corsetti, and P. Nath, in Proceedings of the Fourth International Heidelberg Conference on Dark Matter in Astro and Particle Physics, Cape Town, South Africa, 2002, hep-ph/0204251; Phys. Rev. D 66, 035003 (2002).

[13] T. Nihei, L. Roszkowski, and R. Ruis de Austri, J. High Energy Phys. 05, 063 (2001); L. Roszkowski, R. Ruis de Austri, 
and T. Nihei, ibid. 08, 024 (2001).

[14] G. F. Giudice, E. W. Kolb, and A. Riotto, Phys. Rev. D 64, 023508 (2001).

[15] S. Davidson, M. Losada, and A. Riotto, Phys. Rev. Lett. 84, 4284 (2000).

[16] G. F. Giudice, E. W. Kolb, A. Riotto, D. V. Semikoz, and I. I. Tkachev, Phys. Rev. D 64, 043512 (2001).

[17] For a review on inflation, see D. H. Lyth and A. Riotto, Phys. Rep. 314, 1 (1999).

[18] T. Moroi and L. Randall, Nucl. Phys. B570, 455 (2000).

[19] R. Allahverdi and M. Drees, Phys. Rev. Lett. 89, 091302 (2002); R. Allahverdi and M. Drees, Phys. Rev. D 66, 063513 (2002).

[20] A. Bottino, V. de Alfaro, N. Fornengo, G. Mignola, and M. Pignone, Astropart. Phys. 2, 67 (1994).

[21] J. McDonald, Phys. Rev. D 43, 1063 (1991).

[22] A. Bottino, F. Donato, N. Fornengo, and S. Scopel, Phys. Rev.
D 63, 125003 (2001).

[23] L. Bergström, J. Edsjö, and P. Ullio, Phys. Rev. Lett. 87, 251301 (2001); D. V. Hooper, hep-ph/0103277; L. Bergstrom, J. Edsjo, P. Gondolo, and P. Ullio, Phys. Rev. D 59, 043506 (1999); V. Mandic, A. Pierce, P. Gondolo, and H. Murayama, hep-ph/0008022.

[24] A. Bottino, F. Donato, N. Fornengo, and S. Scopel, Phys. Rev. D 63, 125003 (2001); A. Bottino, N. Fornengo, and S. Scopel, in Proceedings of COSMO-01, Rovaniemi, Finland, 2001, hep-ph/0112238, [Nucl. Phys. B (Proc. Suppl.) 110, 76 (2002)].

[25] N. Fornengo, in Proceedings of Topics in Astroparticle and Underground Physics" (TAUP 2001) [1], p. 26, hep-ph/0201156.

[26] N. Fornengo, in Proceedings of "Sources and Detection of Dark Matter and Dark Energy in the Universe (DM2002)" [2], hep-ph/0206092. 